anti-SSB were absent in the 4 patients. Biopsy of accessory salivary glands revealed the presence of a grade 4 lymphocytic sialadenitis according to the Chisholm and Masson classification in 3 patients, while the remaining case had a lymphocyte grade 2 sialadenitis. The evaluation of hepatic fibrosis did not reveal cirrhosis in the 4 cases.

Conclusion: The prevalence of Sicca syndrome during hepatitis $C$ is estimated at $8 \%$ in our study. It was mainly reported in women with perimenopausal age and seems to be associated with cryoglobulinemia, a high viral load and and an advanced fibrosis.

Disclosure of Interests: None declared

DOI: 10.1136/annrheumdis-2019-eular.7584

\section{AB0530 THE EFFECT OF MYCOPHENOLATE MOFETIL ON NON- RENAL MANIFESTATIONS IN SYSTEMIC LUPUS ERYTHEMATOSUS FROM 2014 TO 2018: OBSERVATION STUDY FROM KOREAN LUPUS NETWORK (KORNET) REGISTRY}

Seong-Kyu Kim ${ }^{1}$, Jung-Yoon Choe $^{2}$, Ui Hong Jung ${ }^{3} .{ }^{1}$ Catholic University of Daegu School of Medicine, Daegu, Korea, Rep. of (South Korea); ${ }^{2}$ Catholic University of Daegu School of Medicine, Daegu, Korea, Rep. of (South Korea); ${ }^{3}$ Daegu Catholic University Medical Center, Daegu, Korea, Rep. of (South Korea)

Background: Mycophenolate mofetil (MMF) has been established to a potent therapeutic drug for regulate renal involvement in systemic lupus erythematosus (SLE).

Objectives: The object of this study is to identify the effect of MMF on non-renal manifestations in SLE.

Methods: The study population was total 439 SLE patients enrolled from Korean Lupus Network (KORNET) registry. The KORNET registry was followed up annually and completed from baseline survey to the $2^{\text {nd }}$ visits from 2014 to 2018. In the subgroup analysis, the effect of MMF on clinical features was also evaluated in 110 patients with histologically confirmed lupus nephritis (LN). The changes of clinical features including mucocutaneous lesions, arthritis, serositis, neurologic disorder, and hematologic abnormalities were assessed between MMF and non-MMF groups during follow-up period. Statistical analysis was used by multiple comparison analysis, considering time, group, and interaction of follow-up time and treatment group.

Results: There was significant difference of malar rash and renal disorder between MMF and non-MMF groups considering time and group in total SLE patients $(p=0.025$ and $p<0.001$, respectively). In hematologic abnormalities, proportion of leukopenia was significantly different between two groups during follow-up periods $\left(p_{\text {time }}<0.001, p_{\text {group }}<0.001\right.$, and $\left.p_{\text {time } \& \text { group }}=0.004\right)$. In $110 \mathrm{LN}$ patients, there was not difference of non-renal clinical features between them. In contrast, proportion of leukopenia in patients with LN was significantly different between two groups during follow-up periods $\left(\mathrm{p}_{\text {time }}<0.001, \mathrm{p}_{\text {group }}=0.003\right.$, and $\mathrm{p}_{\text {time }}$ \& group $=0.004$ ).

Conclusion: This study showed that MMF might be beneficial to treatment for hematologic abnormalities in SLE.

\section{REFERENCES}

[1] Yahya F, Jasmin R, Ng CT, Cheah TE, Sockalingam S. Open label randomized controlled trial assessing the efficacy of mycophenolate sodium against other conventional immunosuppressive agents in active systemic lupus erythematosus patients without renal involvement. Int J Rheum Dis. 2013 Dec;16(6):724-30.

[2] Ginzler EM, Wofsy D, Isenberg D, Gordon C, Lisk L, Dooley MA; ALMS Group. Nonrenal disease activity following mycophenolate mofetil or intravenous cyclophosphamide as induction treatment for lupus nephritis: findings in a multicenter, prospective, randomized, open-label, parallel-group clinical trial. Arthritis Rheum. 2010 Jan;62(1):211-21.

[3] Pisoni CN, Sanchez FJ, Karim Y, Cuadrado MJ, D'Cruz DP, Abbs IC, Khamasta MA, Hughes GR. Mycophenolate mofetil in systemic lupus erythematosus: efficacy and tolerability in 86 patients. J Rheumatol. 2005 Jun;32 (6):1047-52.

[4] Moder KG, Amin S, Mazlumzadeh M, Crowson C, Ytterberg S. The effect of mycophenolate mofetil on patients with active non-renal SLE. Clin Exp Rheumatol. 2007 Nov-Dec;25(6):932.

Acknowledgement: none

Disclosure of Interests: None declared

DOI: 10.1136/annrheumdis-2019-eular.5268

\section{AB0531 \\ OBESITY INCREASES THE INCIDENCE OF NEW-ONSET LUPUS NEPHRITIS AND ORGAN DAMAGE DURING FOLLOW-UP IN PATIENTS WITH SYSTEMIC LUPUS ERYTHEMATOSUS}

Shin-Seok Lee, Ji-Hyoun Kang, Sung-Eun Choi, Haimuzi Xu, Dong-Jin Park. Cheonnam National University Hospital, Gwangju, Korea, Rep. of (South Korea)

\section{Background:}

Objectives: This study investigated the detrimental effects of obesity on clinical manifestations, disease activity, and organ damage in Korean patients with systemic lupus erythematosus (SLE).

Methods: We obtained data on 393 SLE patients from the Korean Lupus Network registry. Demographic variables, clinical manifestations, laboratory findings, Physician Global Assessment (PGA), Systemic Lupus Erythematosus Disease Activity Index (SLEDAI)-2000 and Systemic Lupus International Collaborating Clinics Group (SLICC) damage index scores were recorded at the time of enrollment. The tests were repeated annually for 3 consecutive years. We divided the patients into groups according to their body mass index (BMI) using the Asia-Pacific classification (normal, $\mathrm{BMI}<23$; overweight, $23 \leq \mathrm{BMI}<25$; obese, $\mathrm{BMI} \geq 25$ ). Univariate and multivariate analyses were performed to assess the impact of obesity on clinical outcomes.

Results: Of the 393 patients, $59(15.0 \%)$ were obese at the time of enrollment. Obese patients had more comorbidities, such as diabetes $(P=0.002)$, hypertension $(P=0.005)$, hyperlipidemia $(P=0.005)$, and pulmonary hypertension $(P=0.036)$ than non-obese patients. Nephritis at enrollment and newly developed nephritis during follow-up were more common in obese patients than in non-obese patients $(P=0.002$ and $P=0.002$, respectively). In addition, obese patients had higher daily and cumulative prednisolone doses $(P=0.010$ and $P=0.010$, respectively) and higher rates of intravenous cyclophosphamide $(P=0.008)$, mycophenolate $(P=0.030)$, tacrolimus $(P=0.007)$, and cyclosporine $(P=0.019)$ use than non-obese patients. Furthermore, the PGA and SLICC damage index scores were higher in obese patients than in non-obese patients $(P=0.017$ and $P=0.039$, respectively) for all 3 consecutive years. In the multivariate analysis, obesity was significantly associated with male gender $(\mathrm{OR}=$ $0.141,95 \% \mathrm{Cl}: 0.047-0.419, P<0.001)$, newly developed nephritis (OR $=$ $2.741,95 \% \mathrm{Cl}: 1.080-6.957, P=0.034$ ), and annual increase in SDI (OR $=2.185,95 \% \mathrm{Cl}: 1.229-3.885, P=0.008)$.

Conclusion: Obese SLE patients had a higher incidence of newly developed nephritis and cumulative organ damage than non-obese patients Therefore, lifestyle modifications, including those aimed at weight loss, should be recommended for these patients to improve their clinical outcomes.

Disclosure of Interests: None declared

DOI: 10.1136/annrheumdis-2019-eular.3430

\section{AB0532 SOLUBLE SIGLEC-5 IS A NOVEL SALIVARY BIOMARKER FOR PRIMARY SJOGREN'S SYNDROME}

Jennifer Lee, Moon Young Kim, Sung-Hwan Park. Seoul St Mary's hospital, College of Medicine, The Catholic University of Korea, Division of Rheumatology, Department of Internal Medicine, Seoul, Korea, Rep. of (South Korea)

Background: Despite advances in the understanding of the pathogenesis disease-specific biomarkers have not been included in the classification criteria for Primary Sjogren's syndrome (pSS).

Objectives: Based on the microarray of peripheral blood mononuclear cell (PBMC) of pSS patients, we aimed to investigate whether sialic acid-binding immunoglobulin-like lectin (siglec)-5 might serve as a biomarker for pSS

Methods: Microarray of PBMCs obtained from 26 pSS patients and 10 healthy control $(\mathrm{HC}) \mathrm{s}$ was performed to screen potential biomarkers for pSS. The concentration of siglec-5 in saliva and sera was determined by ELISA. Clinical parameters related with pSS were obtained from pSS registry and correlation with salivary siglec-5 level was evaluated. Receiver operating curve (ROC) analysis was performed to determine cut off value. A separate validation cohort consisted of subjects with suspicious pSS was evaluated to determine the performance.

Results: The level of salivary siglec-5/14 was significantly higher in pSS patients $(n=170)$ compared with HCs $(n=25)$ or non SS sicca patients $(\mathrm{n}=78)(1346.8[202.8-4280.0] \mathrm{pg} / \mathrm{mL}, 6.08$ [0-134.0] $\mathrm{pg} / \mathrm{mL}$, and $195[0$ 947.5] $\mathrm{pg} / \mathrm{mL}$, median [interquartile range], $P<0.001)$, meanwhile the serum level was not different between the groups. Clinical parameters were available in 170 patients in pSS registry. Salivary siglec-5 level negatively correlated with salivary flow rate (spearman's rho: $-0.420, P<0.001$ ), and positively correlated with ocular surface score (rho: $0.331, P<0.001$ ) and serum immunoglobulin $\mathrm{G}$ level (rho $=0.202, \mathrm{P}=0.008$ ). However, the level 